\title{
Acute leukemias in Piauí: comparison with features observed in other regions of Brazil
}

M.F.N. Rego ${ }^{1}$, G.S. Pinheiro ${ }^{1}$, K. Metze ${ }^{2}$ and I. Lorand-Metze ${ }^{3}$

\section{Correspondence}

I. Lorand-Metze

Hemocentro, UNICAMP

Caixa Postal 6198

13083-970 Campinas, SP

Brasil

Fax: +55-19-3788-8600

E-mail: ilmetze@unicamp.br

Publication supported by FAPESP.

Received May 24, 2002

Accepted November 25, 2002
Departamento de Clínica Médica, Faculdade de Medicina, Universidade Federal do Piauí, Teresina, PI, Brasil

Departamentos de ${ }^{2}$ Patologia, and ${ }^{3}$ Clínica Médica,

Faculdade de Ciências Médicas, Universidade Estadual de Campinas, Campinas, SP, Brasil

\begin{abstract}
Differences in age and sex distribution as well as FAB (FrenchAmerican-British classification) types have been reported for acute leukemias in several countries. We studied the demographics and response to treatment of patients with acute myeloid leukemia (AML) and acute lymphoblastic leukemia (ALL) between 1989 and 2000 in Teresina, Piauí, and compared these results with reports from Brazil and other countries. Complete data concerning 345 patients (230 ALL, 115 AML) were reviewed. AML occurred predominantly in adults $(77 \%)$, with a median age of 34 years, similar to that found in the southeast of Brazil but lower than the median age in the United States and Europe (52 years). FAB distribution was similar in children and adults and FAB-M2 was the most common type, as also found in Japan. The high frequency of FAB-M3 described in most Brazilian studies and for Hispanics in the United States was not observed. Overall survival for adults was $40 \%$, similar to other studies in Brazil. A high mortality rate was observed during induction. No clinical or hematological parameter influenced survival in the Cox model. ALL presented the characteristic peak of incidence between 2-8 years. Most of the cases were CD10+ pre-B ALL. In 25\%, abnormal expression of myeloid antigens was observed. Only $10 \%$ of the patients were older than 30 years. Overall survival was better for children. Age and leukocyte count were independent prognostic factors. These data demonstrate that, although there are regional peculiarities, the application of standardized treatments and good supportive care make it possible to achieve results observed in other countries for the same chemotherapy protocols.
\end{abstract}

\section{Introduction}

Geographical variations have been described for age and sex distribution as well as FAB (French-American-British classification) subtypes of acute leukemias, possibly due to ethnic and environmental factors. Acute lymphoblastic leukemia (ALL), the
Key words

- Acute lymphoblastic leukemia

- Acute myeloid leukemia

- Epidemiology most common type of acute leukemia in childhood, has its peak of incidence between 1 and 4 years in most countries (1). The most common phenotype is CD10+ pre-B ALL (1$3)$. The frequency of T-ALL increases with age. Children show a better response to treatment than adults (1).

Regional variations have been described 
for acute myeloid leukemia (AML). FABM2 is the most common type of AML in Japan and its frequency is higher in children than in adults (4), a fact that was not observed in Australia (5). Promyelocytic leukemia (FAB-M3) shows a remarkably high frequency among Hispanics in the United States (6) and in several countries in South America including Brazil (7-9).

Brazil is a large country with striking differences in climate, ethnic heterogeneity and degree of industrialization. In the present study we describe the demographic features and response to treatment of acute leukemias (children and adults, ALL and AML) observed at a large regional hospital in Teresina, PI, northern Brazil, which represents the only cancer center for a region of about 3 million people. We compared several of these features with results described for other regions of Brazil, especially Campinas, located in the subtropical, highly industrialized southeast.

\section{Patients and Methods}

All newly diagnosed patients with ALL and AML treated at the São Marcos Hospital (the only hospital that treats acute leukemias in the region), Teresina, PI, from January 1989 to December 2000 were enrolled in this retrospective study. Teresina is the capital of a region with 3 million inhabitants located at $5^{\circ}$ latitude south. Exclusion criteria were the absence of complete medical records or bone marrow smears for review. Secondary leukemias (cases with a past history of chemotherapy or radiotherapy or myelodysplasia) and chronic myeloid leukemia in blast crisis were also excluded.

The following parameters were collected for each patient: age, sex, presence of fever and organomegalies, body mass index (weight/ height squared) and peripheral blood counts. The diagnosis of the type of leukemia was made according to the guidelines of the International Council for Standardization in
Haematology (10): a diagnosis of acute leukemia was made when bone marrow blasts were above $30 \%$. Bone marrow smears were stained with Wright-Giemsa, periodic acidSchiff, Sudan black, and alpha-naphthyl-acetate esterase, $\mathrm{pH}$ 7.4. Cases with blasts negative for Sudan black and alpha-naphthyl-acetate esterase were considered to be ALL. All smears were reviewed and classified according to FAB criteria. Since 1998, immunophenotyping was performed in childhood ALL patients using monoclonal antibodies to the following antigens: CD20, CD22, CD10, cytoplasmic IgM, CD2, CD3, CD5, CD7, CD34, HLA-DR, CD13, CD14, $\mathrm{CD} 15, \mathrm{CD} 33$ and myeloperoxidase. Immunophenotyping was carried out by flow cytometry using a FACscan apparatus and 2color routine procedures.

Overall survival was calculated by the Kaplan-Meier method for all patients according to date of diagnosis and date of death or last follow-up.

Patients wit ALL aged less than 18 years were treated according to the Brazilian Protocol for ALL (11). Adults with ALL were treated according to the German Protocol (12). Children with AML were treated according to the BFM-83 study (13) and adults received $150 \mathrm{mg} / \mathrm{m}^{2}$ cytosine arabinoside (araC) for 7 days and $50 \mathrm{mg} / \mathrm{m}^{2}$ daunorubicin for 3 days followed by high dose ara-C $(9,14)$.

The data on AML were compared with a monoinstitutional study of patients from Campinas, SP, southeast region (15), treated according to the TAD9 Protocol (using araC, daunorubicin, 6-thioguanin) from the German AML Study Group.

The project was approved by the Hospital Ethics Committee.

\section{Statistical analysis}

First, descriptive statistics were applied to all the parameters analyzed. The frequencies of FAB types in Teresina and Campinas were compared by the chi-square test. Sur- 
vival was analyzed by the Kaplan-Meier method. Univariate and multivariate analysis for the features influencing survival in ALL and AML was performed according to the proportional hazard model of Cox (16).

\section{Results}

\section{Acute myeloid leukemia}

Complete charts and smears were retrieved for 115 patients. Their clinical features are shown in Table 1. The majority of the patients were adults (Figure 1). The male: female ratio was similar for children and adults. Only 9 patients (4 children and 5 adults) had a low body mass index. The FAB subtypes of the patients are shown in Table 2 compared to those found in the study from Campinas (15). A similar distribution of FAB subtypes was found in children and adults in Teresina $(P=0.20)$. The most common type was FAB-M2 (32.8\%), followed by FABM4 and FAB-M5 (15.6\% each). Among the patients from Campinas, the most common type was FAB-M4 (32.4\%) followed by FABM3 (21.1\%). These differences between Campinas and Teresina were statistically significant $(\mathrm{P}<0.0001)$.

Overall survival was $40 \%$ among the patients from Teresina, including children and adults. There was no difference in survival according to age (Figure 2). However, 20 patients $(17 \%)$ died within the first 2 months after diagnosis. In univariate analysis, none of the parameters analyzed showed an influence on survival. However, when the patients were stratified for FAB subtype there was a positive association between subtype and age.

When the results of chemotherapy (Figure 3 ) were compared with those for the cases from the Campinas study (age-matched), no difference in outcome was observed $(\mathrm{P}=0.22)$.

\section{Acute lymphoblastic leukemia}

During the study period, 230 cases were
Table 1. Clinical features of the patients with acute myeloid leukemia from Teresina.

\begin{tabular}{lc}
\hline Age (years) & $34(0.8-80)$ \\
Age distribution & \\
$0-15$ years & 19 \\
$11-15$ years & 7 \\
16-60 years & 62 \\
$>60$ years & 27 \\
Sex (male/female) & \\
$0-15$ years & $16 / 10$ \\
$>15$ years & $49 / 40$ \\
Fever & 66 \\
Hepatomegaly & 16 \\
Splenomegaly & 18 \\
Enlarged lymph nodes & 9 \\
Hematocrit $(\%)$ & $20(6-43)$ \\
Leukocytes $\times 10^{9} / 1$ & $12.6(0.6-76.0)$ \\
Platelets $\times 109 / 1$ & $29(1-295)$
\end{tabular}

Data are reported as median (range) or number of patients.

Table 2. Comparison of $F A B$ subtypes of acute myeloid leukemia patients from Campinas and Teresina.

\begin{tabular}{lcc} 
FAB subtype & Campinas (\%) & Teresina (\%) \\
\hline M0 & 1.0 & 3.1 \\
M1 & 15.6 & 13.9 \\
M2 & 20.1 & 32.8 \\
M3 & 21.1 & 7.8 \\
M4 & 32.4 & 15.6 \\
M5 & 5.6 & 15.6 \\
M6 & 5.1 & 3.1 \\
M7 & 0.5 & 1.5
\end{tabular}

Table 3. Clinical characteristics of the patients with acute lymphoblastic leukemia.

\begin{tabular}{lc}
\hline Age (years) & $8.5(0.9-85)$ \\
Age distribution & \\
$\quad 0-10$ years & 135 \\
$10-18$ years & 44 \\
$>18$ years & 51 \\
Sex (male/female) & \\
$\quad<15$ years & $95 / 64$ \\
$15-18$ years & $14 / 2$ \\
$>18$ years & $32 / 23$ \\
Fever & 137 \\
Enlarged lymph nodes & 130 \\
Hepatomegaly & 106 \\
Splenomegaly & 122 \\
Hematocrit $(\%)$ & $22(6-48)$ \\
Leukocytes $\times 10^{9} / 1$ & $18.6(0.3-95)$ \\
Platelets $\times 10 \% /$ & $36(2-390)$
\end{tabular}

Data are reported as median (range) or number of patients. 
treated in Teresina. Their clinical features are presented in Table 3. Most patients (77\%) were less than 18 years old (Figure 4) and were treated according to the Brazilian Protocol for Childhood ALL (10). There was a predominance of males among both children and adults. Organomegaly was very frequent. In 65 children $(36 \%)$ the leukocyte count

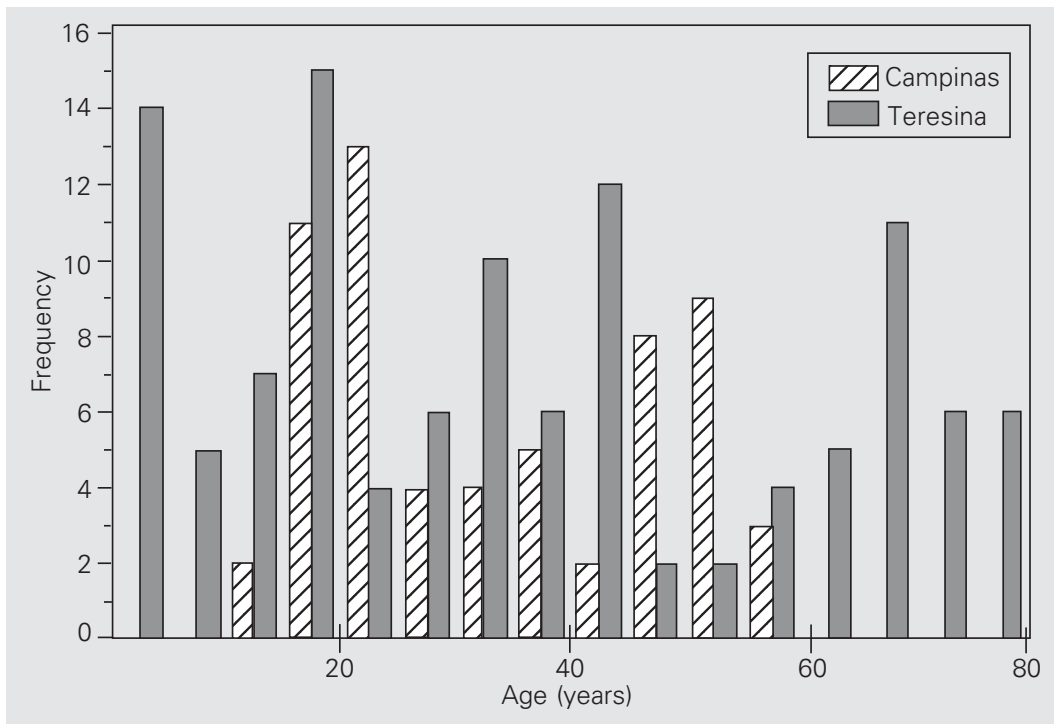

Figure 1. Age distribution of the patients with acute myeloid leukemia in Teresina compared to that of the Campinas study.

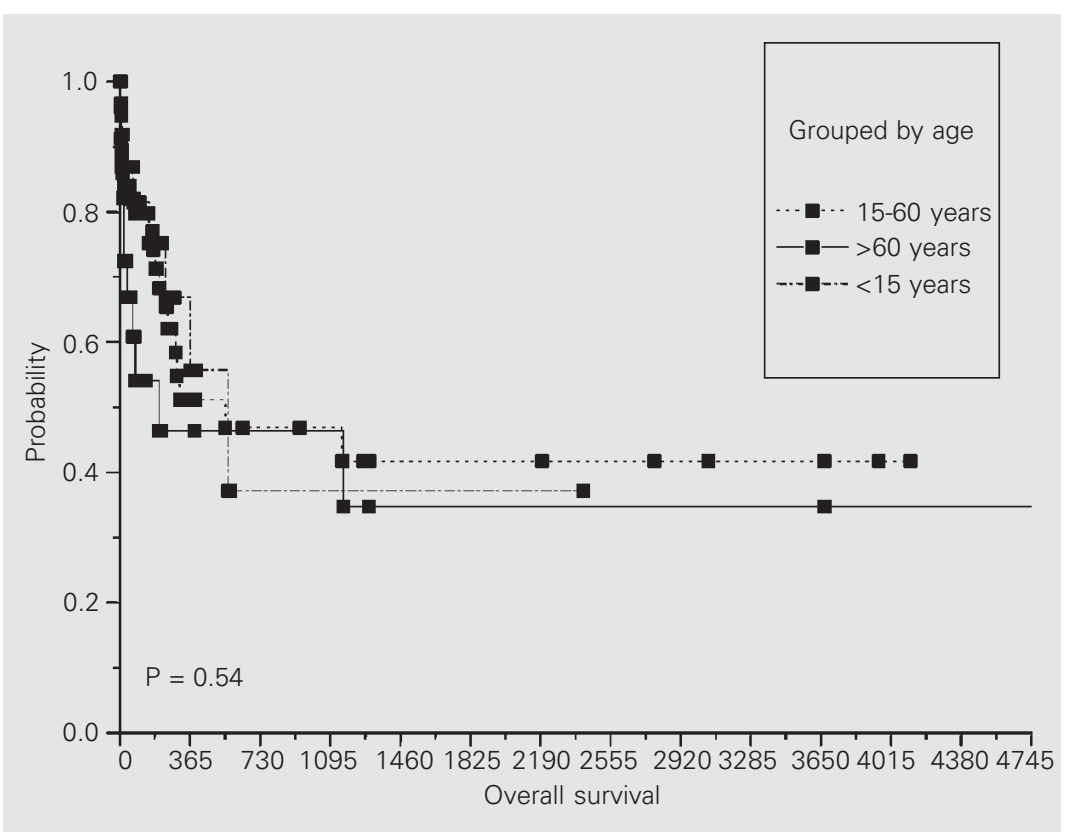

Figure 2. Overall survival of the patients with acute myeloid leukemia in Teresina. Children ( $<15$ years), adults (15-60 years), and patients $>60$ years are treated as separate groups. was above $50 \times 10^{9} / 1$. A low body mass index was observed in only 11 cases.

Immunophenotyping was performed in 31 cases. Among them, 26 were of the B-cell lineage ( 24 of them $\mathrm{CD} 10+$ ) and 5 were $\mathrm{T}$ ALL. Abnormal expression of myeloid antigens was observed (CD13 and/or CD33) in one T-ALL and in 7 B-derived ALL cases.

Survival analysis demonstrated a better survival for children than for adults (Figure 5 ), with a 10 -year survival rate of $52 \%$ for the former. Mortality during induction was $11 \%$. In univariate analysis the peripheral leukocyte count at diagnosis and patient age showed an influence on survival $(R=0.1$, $\mathrm{P}=0.002$ and $\mathrm{R}=0.11, \mathrm{P}=0.0015$, respectively). In multivariate analysis, both parameters remained significant.

\section{Discussion}

This retrospective study covered a period of eleven years and the results are representative for the entire region of Teresina, Piauí. This region is located in the north of Brazil, at the Equator, in an agricultural region with a low per capita income. However, the São Marcos Hospital is well equipped for chemotherapy and supportive care. During the study period, the patients with ALL were almost twice the number of patients with AML.

AML was predominant in adults, with a median age lower than that observed for the United States (6) but similar to that observed in other Brazilian studies $(9,15,17,18)$. The incidence of AML increases with age and is more frequent above 60 years (18). This is probably due to a higher life expectancy and increasing environmental exposure to carcinogens. Life expectancy is lower in Brazil, especially in the north and northeast regions when compared to Europe or the United States. Existing epidemiological data refer to a lower incidence of AML in Brazil. This may be more probably due to the absence of the peak of incidence among old people observed in developed countries than to 

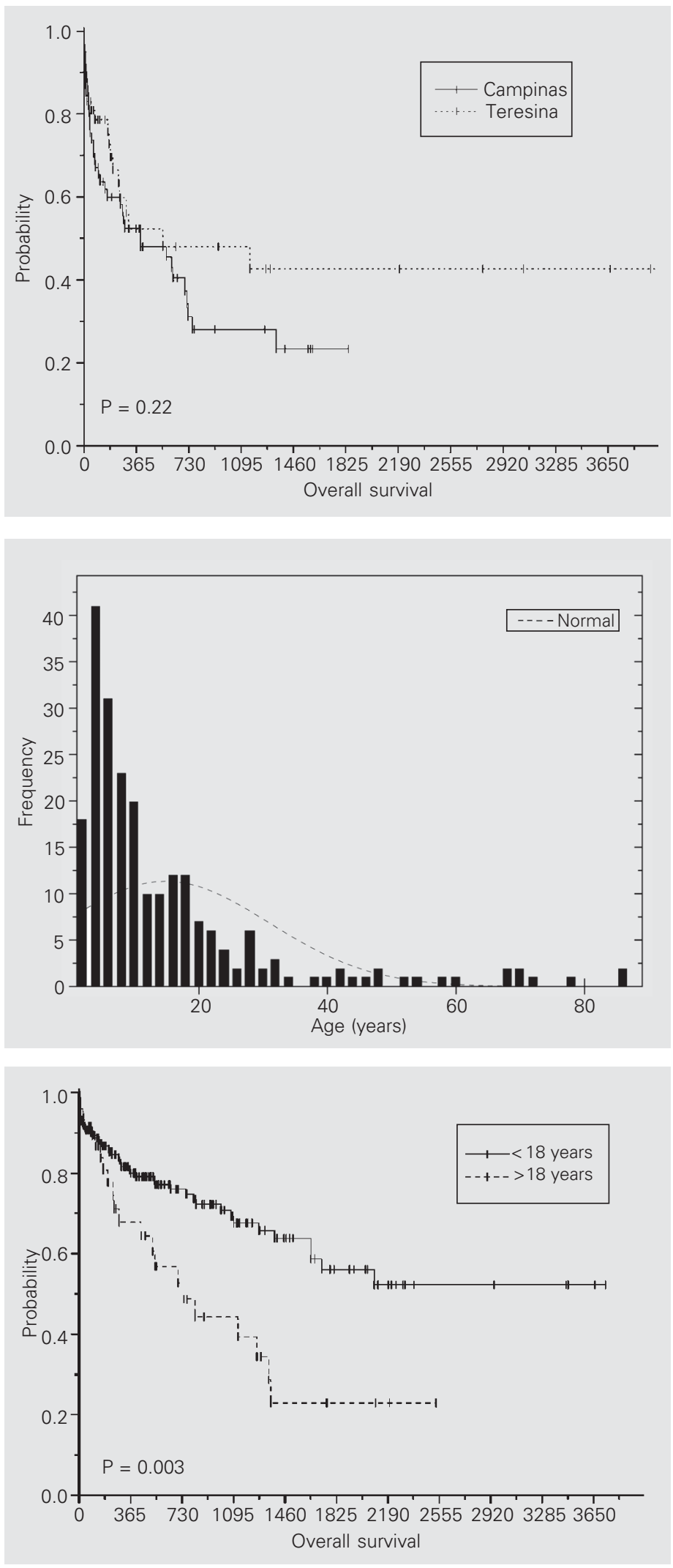

Figure 3. Comparison of the survival of patients from Teresina and Campinas with acute myeloid leukemia. Although a high mortality rate was observed during the first 2 months, those achieving complete remission showed the survival behavior usually described for the protocols used.

Figure 4. Age distribution of the acute lymphoblastic leukemia patients. There was a predominance of children, with a peak between 2 and 8 years. Most cases were below 30 years.

Figure 5. Survival of patients with acute lymphoblastic leukemia Children had a significant better survival than adults. 
underdiagnosis, as stated by Fagundes (17). Likewise, also myelodysplastic syndromes in Brazil occur at a younger age than in these countries (19).

The distribution of FAB subtypes was similar in children and adults. Since in Teresina no immunophenotyping was performed in adult leukemia patients, some cases of AML FABM0 were probably misdiagnosed. But as the expected frequency of this subtype is low, it did not significantly change the relative frequency of the more common FAB types. However, a low proportion of FAB-M3 was observed. This is uncommon for what has been described in other regions of Brazil $(8,9,15,17$, 18,20 ), in Latin America (7) or among Hispanics in the United States (6). Although no cytogenetic diagnosis was performed, the morphological diagnosis of promyelocytic leukemia has been considered highly reliable and reproducible $(6,21)$. Therefore, one may speculate that perhaps the patients with promyelocytic leukemia may have died from bleeding before they reached the reference center for treatment. TheFAB-type distribution between Campinas (southeast) and Teresina (north) also presented differences. In the former, FAB-M4 was more common, whereas in the latter FABM2 was predominant. A similar discrepancy was observed in the study by Nakase et al. (5) where FAB-M2 was more frequent in Japanese patients and FAB-M4 was more common in Australia. As the concentration of Amerindians is higher in the north of Brazil than in the southeast (22), one may speculate that AML FAB-M2 is more common in Orientals and Amerindians. One could also suppose that in Piauí, an essentially agricultural region, people are exposed to different carcinogens than in the southeast, where industrial genotoxins are present. Therefore, the ethnic composition and environmental factors could play a role in this distribution. Although a high mortality was observed during induction treatment, the overall survival of the patients achieving complete remission was good and similar to that observed in other regions of Brazil
$(9,15,17)$. A high mortality rate during induction therapy was also found in these studies, mainly due to infection. A high frequency of fever and infection was observed in these patients at diagnosis, explaining the poor performance obtained during induction therapy.

Most patients with ALL were children, and even most of the cases considered as "adults" were below 30 years old. A peak of incidence between 2-10 years was found, as observed in most studies around the world but not in Africa (23). This pattern of incidence has been well established but has only recently emerged among African American and Japanese children $(1,4)$. Only few cases were older than 30 years, in contrast to the United States and Europe, where a small peak of incidence emerges above 50 years $(1,5)$. Most cases where immunophenotyping was performed were common ALL (CD19/CD10+). Also, the pattern of aberrant expression of myeloid antigens was similar to that observed in other studies (1-3). Age and peripheral leukocyte counts were the most important factors influencing outcome in the patients.

Children were treated according to the Brazilian Protocol for Childhood ALL, a nationwide study for ALL treatment that has been successful and up-to-date, permitting the participating centers to obtain treatment results comparable to those observed in other countries. The survival rate, however, was below what was expected for this protocol. Mortality during induction was low, but several patients died during maintenance treatment or relapsed. This is in agreement with that expected for patients with a poor socioeconomic status, among whom tolerance of and compliance with maintenance treatment are impaired, as stated by Viana et al. (24).

We conclude from these results that AML shows more geographical variation than ALL. Furthermore, the use of state-of-the art protocols and good supportive care are important for achieving a successful treatment outcome. 


\section{References}

1. Mauer AM (1993). Adult and childhood lymphocytic leukemia: are they different diseases? American Journal of Hematology, 42: 127131.

2. Khalidi HS, Chang KL, Medeiros J, Brynes RK, Slovak ML, MurataCollins JL \& Arber DA (1999). Acute lymphoblastic leukemia: survey of immunophenotype, FAB classification, frequency of myeloid antigen expression and karyotypic abnormalities in 210 pediatric and adult cases. American Journal of Clinical Pathology, 111: 467-476.

3. Paredes-Aguillera R, Romero-Guzman L, Lopez-Santiago N, BurbanoCeron L, Camacho-del Monte O \& Nieto-Martinez S (2001). Flow cytometric analysis of cell-surface and intracellular antigens in the diagnosis of acute leukemia. American Journal of Hematology, 68: 69-74.

4. Horibe K, Tsukimoto I \& Ohno R (2001). Clinicopathologic characteristics of leukemia in Japanese children and young adults. Leukemia, 15: 1256-1261.

5. Nakase K, Bradstock K, Sartor M, Gottlieb D, Byth K, Shiku H, Kamada N \& The Japanese Cooperative Group of Leukemia/Lymphoma (2000). Geographic heterogeneity of cellular characteristics of acute leukemia: a comparative study of Australian and Japanese adult cases. Leukemia, 14: 163-168.

6. Douer D, Preston-Martin S, Chang E, Nichols PW, Watkins KJ \& Levine AM (1996). High frequency of acute promyelocytic leukemia among latinos with acute myeloid leukemia. Blood, 87: 308-313.

7. Otero JC, Santillana S \& Fereyros G (1996). High frequency of acute promyelocytic leukemia among latinos with acute myeloid leukemia. Blood, 88: 377-379.

8. Loureiro P, Azevedo A, Maia A, Freire AD, Souto F, Bandeira F, Vasconcelos J \& Paiva A (1992). Acute myeloid leukemia: presentation of the disease and response to treatment in Northeast Brazil. Medical and Pediatric Oncology, 20: 439.

9. Fagundes EM, Rocha VG, Azevedo WM, Clementino NCD, Quintão JS, Ferraz MHC \& Viana MB (1995). Leucemia mieloide aguda do adulto: análise retrospectiva de 99 casos. Boletim da Sociedade Brasileira de Hematologia e Hemoterapia, 17: 33-39.

10. Scott CS, Ottolander GJD, Swirsky D et al. (1995). Recommended procedures for the classification of acute leukemias. Leukemia and Lymphoma, 18 (Suppl 1): 1-12.

11. Brandalise $S$, Odone $V$, Pereira W, Andrea M, Zanichelli M \& Aranega $V$ (1993). Treatment results of three consecutive Brazilian cooperative childhood ALL protocolos: GBTLI-80, GBTLI-82 and -85. ALL Brazilian Group. Leukemia, 7 (Suppl 2): S142-S145.

12. Hoelzer D, Ludwig WD, Thiel E et al. (1996). Improved outcome in adult B-cell acute lymphoblastic leukemia. Blood, 87: 495-508.

13. Sartori PCE, Taylor MH, Stevens MCG, Darbyshire PJ \& Mann JR (1993). Treatment of childhood acute myeloid leukaemia using the BFM-83 protocol. Medical and Pediatric Oncology, 21: 8-13.

14. Yates JW, Wallace Jr HT, Ellison RR \& Holland JF (1973). Cytosine arabinoside and daunorubicin therapy in acute non-lymphocytic leukemia. Cancer Chemotherapy Reports, 57: 485-488.

15. Pagnano KB, Traína F, Takahashi T, Oliveira GB, Rossini MS, LorandMetze I, Vigorito AC, Miranda ECM \& Souza CA (2000). Conventional chemotherapy for acute myeloid leukemia: a Brazilian experience. São Paulo Medical Journal, 118: 173-178.

16. Cox DR (1972). Regression models and life tables. Journal of the Royal Statistical Society, Series B: Statistical Methods, 34: 187-195.

17. Fagundes EM (2002). AML in Brazil: can we improve our treatment results? Série de Monografias da Escola Brasileira de Hematologia, 9: 107-116.

18. Fagundes EM (2002). Prognostic factors in adult acute myeloid leukemia. Série de Monografias da Escola Brasileira de Hematologia, 9: 66-76.

19. Magalhães SMM, Rocha Filho FD, Vassallo J, Pinheiro MP, Metze K \& Lorand-Metze | (2002). Bone marrow lymphoid aggregates in myelodysplastic syndromes. Leukemia Research, 26: 525-530.

20. Martins SRL, Rego EM \& Falcão RP (2001). A classificação das leucemias agudas: citologia, citoquímica e imunofenotipagem. In: Zago MA, Falcão RP \& Pasquini R (Editors), Hematologia: Fundamentos e Prática. Atheneu, São Paulo, SP; Rio de Janeiro, RJ, and Belo Horizonte, MG, Brazil.

21. Chauffaille MLLF, Figueiredo MS, Beltrani R, Antunes SV, Yamamoto M \& Kerbauy J (2001). Acute promyelocytic leukemia: the study of $\mathrm{t}(15 ; 17)$ translocation by fluorescent in situ hybridization, reverse transcriptase-polymerase chain reaction and cytogenetic techniques. Brazilian Journal of Medical and Biological Research, 34: 735-743.

22. Alves-Lima J, Silva-Santos M, Guimarães PEM, Ferreira ACS, Bandelt HJ, Pena SDJ \& Prado VF (2000). The ancestry of Brazilian mtDNA lineages. American Journal of Human Genetics, 67: 444-461.

23. Williams CKO, Folami AO, Laditan AAO \& Ukaejhofo EO (1982). Childhood acute leukemia in a tropical population. British Journal of Cancer, 46: 89-94.

24. Viana MB, Fernandes RAF, Oliveira BM, Murao M, Paes CA \& Duarte AA (2001). Nutritional and socio-economic status in the prognosis of the childhood lymphoblastic leukemia. Haematologica, 86: 113-120. 